\title{
Spin effects on the dynamics of compact binaries
}

\author{
Jan Steinhoff \\ Max Planck Institute for Gravitational Physics (Albert Einstein Institute) \\ Am Mühlenberg 1, D-14476 Potsdam-Golm, Germany \\ E-mail: jan.steinhoff@aei.mpg.de
}

\begin{abstract}
Compact binaries are the most promising source for the advanced gravitational wave detectors, which will start operating this year. The influence of spin on the binary evolution is an important consequence of general relativity and can be large. It is argued that the spin supplementary condition, which is related to the observer dependence of the center, gives rise to a gauge symmetry in the action principle of spinning point-particles. These spinning point-particles serve as an analytic model for extended bodies. The internal structure can be modelled by augmenting the point-particle with higher-order multipole moments. Consequences of the recently discovered universal (equation of state independent) relations between the multipole moments of neutron stars are discussed.
\end{abstract}

Keywords: binary systems; gravitational waves; spin; MG14 Proceedings

\section{Conservative Spin Effects to Fourth Post-Newtonian Order}

An important source for the advanced ground-based gravitational wave detectors ${ }^{1-3}$ are inspiraling and merging binaries of compact stellar-mass objects. An analytic description of the inspiral phase and the emitted gravitational waves is given by the post-Newtonian (PN) approximation, which is a weak field and slow motion approximation. The description of the conservative part of the motion was completed to $4 \mathrm{PN}$ order for (nonrotating) point masses recently. ${ }^{4,5}$

However, it is important to include the effects of the angular momenta of the bodies, i.e., theirs spins, to the same order of approximation. Also this was achieved recently. All the work that went into this can be summarized in the following table, sorted by PN and spin order S:

\begin{tabular}{|c|c|c|c|c|c|c|}
\hline & $1.5 \mathrm{PN}$ & $2 \mathrm{PN}$ & $2.5 \mathrm{PN}$ & $3 \mathrm{PN}$ & $3.5 \mathrm{PN}$ & $4 \mathrm{PN}$ \\
\hline \multirow{2}{*}{$\mathrm{S}$} & $\mathrm{H}^{6-10}$ & & $\mathrm{H}^{11-15}$ & & $\mathrm{H}^{16-18}$ & \\
\hline & $\mathrm{E}^{19,20}$ & & $\mathrm{E}^{21,22}$ & & $\mathrm{E}^{23,24}$ & \\
\hline \multirow{2}{*}{$\mathrm{S}_{1}^{2}$} & & $\mathrm{H}^{7,8,25}$ & & $\mathrm{H}^{26-28}$ & & $\mathrm{H}^{29,30}$ \\
\hline & & $\mathrm{E}^{19,20}$ & & $E^{31}$ & & \\
\hline \multirow{2}{*}{$\mathrm{S}_{1} \mathrm{~S}_{2}$} & & $\mathrm{H}^{7,8}$ & & $\mathrm{H}^{32-34}$ & & $\mathrm{H}^{17,35,36}$ \\
\hline & & $\mathrm{E}^{19,20}$ & & $\mathrm{P}^{37} \mathrm{E}^{31}$ & & \\
\hline \multirow{2}{*}{$\mathrm{S}^{3}$} & & & & & $\mathrm{H}^{38-41}$ & \\
\hline & & & & & $E^{41} P^{42}$ & \\
\hline \multirow{2}{*}{$\mathrm{S}^{4}$} & & & & & & $\mathrm{H}^{40}$ \\
\hline & & & & & & $\mathrm{P}^{38,39,42}$ \\
\hline : & & & & & & $\ddots$ \\
\hline
\end{tabular}


Here $\mathrm{H}$ stands for Hamiltonians or potentials, E refers to results for equations of motion, and $\mathrm{P}$ denotes partial/incomplete results. Some of the references at $S_{1}^{2}$ order are only valid for black holes but not for generic bodies like neutron stars. ${ }^{19,27,38,39}$

A variety of different formalisms was used, in particular for the more recent works. ${ }^{30,33,43,44}$ This is important, since the calculations bear conceptual and technical difficulties, which makes independent checks mandatory. For instance, a disagreement in the $4 \mathrm{PN}$ point-mass results ${ }^{4,5}$ still needs to be reconciled. In the case of spinning bodies, some works were dedicated to establish connections between the formalisms and the comparison of results. ${ }^{45,46}$ All results to $4 \mathrm{PN}$ with spin were checked by independent collaborations and using independent methods, except for the next-to-next-to-leading order $S_{1}^{2}$ potential. ${ }^{29}$ (The $S^{4}$ partial result ${ }^{38,39,42}$ taken together form a complete result in agreement with Ref. 40.) It is also the only result where a Hamiltonian form is missing, from which, e.g., the gauge invariant binding energy can be derived.

\section{Spin Gauge Symmetry in an Action Approach}

It is convenient to derive the above results from an action principle. For a review of action principles with spin, see, e.g., Refs. 43, 47. A more recent concept is the introduction of a so called spin gauge symmetry at the level of the action. ${ }^{30,48}$ The motivation for this symmetry arises from the observer-dependence of the center in relativity. This is best explained by the following figure.

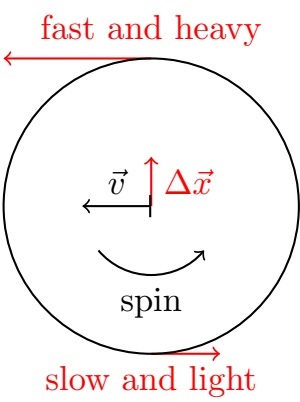

This ambiguity in the center becomes problematic once a quadrupole or higher multipoles of the body are taken into account, since the definition of the multipoles hinges on the center as a reference point. One way to overcome this problem is to pick the rest-frame center of mass, which is singled out since the rest frame provides an intrinsically defined observer. But other centers are useful, too, for instance for Hamiltonian descriptions. Now, any choice of a reference point in a body describes the same physical situation, i.e., it can be understood as a gauge choice. It is then natural to expect that this gauge freedom should correspond to a (gauge) symmetry in an action principle, as implemented in Refs. ${ }^{30,48}$. Interestingly, this action is supported on the rest-frame center worldline and the shift is encoded through a 
time derivative of the linear momentum in the action.

The choice of center is usually encoded in a condition on the spin 4-tensor. This is called the spin supplementary condition. A generalized version of this condition is the generator of spin gauge transformations in the special relativistic case. ${ }^{48}$

\section{Finite Size, Multipole Moments, and Universal Relations}

The (effective) action principle for spinning bodies is a point-particle action with support on a worldline. An interesting question is therefore how the finite size of the body is taken into account. On large scales, the internal structure is encoded in the multipole moments of the bodies. These are represented in the action through nonminimal coupling terms. Following an effective field theory philosophy, one can construct all possible terms compatible with the symmetries, with a constant for each term. The first terms in this expansion indeed correspond to the quadruple, octupole, and hexadecapole $26,30,41,42$.

If all the constants in the action would be arbitrary, then one obtains an undesirable enlargement of the parameter space for waveform models. Fortunately, certain universal relations were discovered ${ }^{50,51}$ for slow rotating compact objects (neutron stars, quark stars), which are approximately independent of the nuclear equation of state. These also hold for rapid rotation ${ }^{52,53}$ and for multipole moments up to the hexadecapole ${ }^{52,54}$. This implies that the waveform model does approximately depend only on one additional constant, which takes on different values for black holes and each neutron star model. This even holds when tidal effects are taken into account. Such a reduction of parameters is important, since the difficulties in extracting parameters from gravitational waves grow significantly with the dimension of the parameter space.

\section{References}

1. http://ligo.org.

2. http://www.virgo-gw.eu.

3. http://gwcenter.icrr.u-tokyo.ac.jp/en.

4. T. Damour, P. Jaranowski, and G. Schäfer, "Nonlocal-in-time action for the fourth post-Newtonian conservative dynamics of two-body systems," Phys. Rev. D89 (2014) 064058, arXiv: 1401.4548 [gr-qc].

5. L. Bernard, L. Blanchet, A. Bohé, G. Faye, and S. Marsat, "Fokker action of non-spinning compact binaries at the fourth post-Newtonian approximation," arXiv:1512.02876 [gr-qc].

6. W. Tulczyjew, "Equations of motion of rotating bodies in general relativity theory," Acta Phys.Polon. 18 (1959) 37-55,534.

7. B. M. Barker and R. F. O'Connell, "Gravitational Two-Body Problem with Arbitrary Masses, Spins, and Quadrupole Moments," Phys. Rev. D12 (1975) $329-335$. 
8. B. M. Barker and R. F. O'Connell, "The gravitational interaction: Spin, rotation, and quantum effects - a review," Gen. Relativ. Gravit. 11 (1979) $149-175$.

9. T. Damour, "Probléme des deux corps et freinage de rayonnement en relativité générale," C. R. Acad. Sci. Paris Sér. II 294 (1982) 1355-1357.

10. T. Damour and G. Schäfer, "Higher Order Relativistic Periastron Advances and Binary Pulsars," Nuovo Cim. B101 (1988) 127-176.

11. T. Damour, P. Jaranowski, and G. Schäfer, "Hamiltonian of two spinning compact bodies with next-to-leading order gravitational spin-orbit coupling," Phys. Rev. D77 (2008) 064032, arXiv:0711.1048 [gr-qc].

12. J. Steinhoff, G. Schäfer, and S. Hergt, "ADM canonical formalism for gravitating spinning objects," Phys. Rev. D77 (2008) 104018, arXiv:0805.3136 [gr-qc].

13. D. L. Perrodin, "Subleading Spin-Orbit Correction to the Newtonian Potential in Effective Field Theory Formalism," in Proceedings of the 12th Marcel Grossmann Meeting on General Relativity, pp. 725-727. World Scientific, Singapore, 2011. arXiv:1005.0634 [gr-qc].

14. R. A. Porto, "Next to leading order spin-orbit effects in the motion of inspiralling compact binaries," Class. Quant. Grav. 27 (2010) 205001, arXiv: 1005.5730 [gr-qc].

15. M. Levi, "Next to Leading Order gravitational Spin-Orbit coupling in an Effective Field Theory approach," Phys. Rev. D82 (2010) 104004, arXiv: 1006.4139 [gr-qc].

16. J. Hartung and J. Steinhoff, "Next-to-next-to-leading order post-Newtonian spin-orbit Hamiltonian for self-gravitating binaries," Annalen Phys. 523 (2011) 783-790, arXiv:1104.3079 [gr-qc].

17. J. Hartung, J. Steinhoff, and G. Schäfer, "Next-to-next-to-leading order post-Newtonian linear-in-spin binary Hamiltonians," Annalen Phys. 525 (2013) 359-394, arXiv:1302.6723 [gr-qc].

18. M. Levi and J. Steinhoff, "Next-to-next-to-leading order gravitational spin-orbit coupling via the effective field theory for spinning objects in the post-Newtonian scheme," JCAP (accepted) (2015), arXiv:1506.05056 [gr-qc].

19. P. D. D'Eath, "Interaction of two black holes in the slow-motion limit," Phys. Rev. D12 (1975) 2183-2199.

20. K. S. Thorne and J. B. Hartle, "Laws of motion and precession for black holes and other bodies," Phys. Rev. D31 (1985) 1815-1837.

21. H. Tagoshi, A. Ohashi, and B. J. Owen, "Gravitational field and equations of motion of spinning compact binaries to 2.5 postNewtonian order," Phys. Rev. D63 (2001) 044006, arXiv:gr-qc/0010014 [gr-qc].

22. G. Faye, L. Blanchet, and A. Buonanno, "Higher-order spin effects in the dynamics of compact binaries. I. Equations of motion," Phys. Rev. D74 
(2006) 104033, arXiv:gr-qc/0605139 [gr-qc].

23. S. Marsat, A. Bohe, G. Faye, and L. Blanchet, "Next-to-next-to-leading order spin-orbit effects in the equations of motion of compact binary systems," Class. Quant. Grav. 30 (2013) 055007, arXiv:1210.4143 [gr-qc].

24. A. Bohe, S. Marsat, G. Faye, and L. Blanchet, "Next-to-next-to-leading order spin-orbit effects in the near-zone metric and precession equations of compact binaries," Class. Quant. Grav. 30 (2013) 075017, arXiv:1212.5520.

25. E. Poisson, "Gravitational waves from inspiraling compact binaries: The Quadrupole moment term," Phys. Rev. D57 (1998) 5287-5290, arXiv:gr-qc/9709032 [gr-qc].

26. R. A. Porto and I. Z. Rothstein, "Next to Leading Order Spin(1)Spin(1) Effects in the Motion of Inspiralling Compact Binaries," Phys. Rev. D78 (2008) 044013, arXiv:0804.0260 [gr-qc]. [Erratum: Phys. Rev.D81,029905(2010)].

27. J. Steinhoff, S. Hergt, and G. Schäfer, "Spin-squared Hamiltonian of next-to-leading order gravitational interaction," Phys. Rev. D78 (2008) 101503, arXiv:0809.2200 [gr-qc].

28. S. Hergt, J. Steinhoff, and G. Schäfer, "Reduced Hamiltonian for next-to-leading order Spin-Squared Dynamics of General Compact Binaries," Class. Quant. Grav. 27 (2010) 135007, arXiv:1002.2093 [gr-qc].

29. M. Levi and J. Steinhoff, "Next-to-next-to-leading order gravitational spin-squared potential via the effective field theory for spinning objects in the post-Newtonian scheme," JCAP (accepted) (2015), arXiv:1506.05794 [gr-qc].

30. M. Levi and J. Steinhoff, "Spinning gravitating objects in the effective field theory in the post-Newtonian scheme," JHEP 09 (2015) 219, arXiv: 1501.04956 [gr-qc].

31. A. Bohé, G. Faye, S. Marsat, and E. K. Porter, "Quadratic-in-spin effects in the orbital dynamics and gravitational-wave energy flux of compact binaries at the 3PN order," Class. Quant. Grav. 32 no. 19, (2015) 195010, arXiv: 1501.01529 [gr-qc].

32. J. Steinhoff, S. Hergt, and G. Schäfer, "On the next-to-leading order gravitational spin(1)-spin(2) dynamics," Phys. Rev. D77 (2008) 081501, arXiv:0712.1716 [gr-qc].

33. R. A. Porto and I. Z. Rothstein, "Spin(1)Spin(2) Effects in the Motion of Inspiralling Compact Binaries at Third Order in the Post-Newtonian Expansion," Phys. Rev. D78 (2008) 044012, arXiv:0802.0720 [gr-qc]. [Erratum: Phys. Rev.D81,029904(2010)].

34. M. Levi, "Next to Leading Order gravitational Spin1-Spin2 coupling with Kaluza-Klein reduction," Phys. Rev. D82 (2010) 064029, arXiv:0802.1508 [gr-qc].

35. J. Hartung and J. Steinhoff, "Next-to-next-to-leading order post-Newtonian 
spin(1)-spin(2) Hamiltonian for self-gravitating binaries," Annalen Phys. $\mathbf{5 2 3}$ (2011) 919-924, arXiv: 1107.4294 [gr-qc].

36. M. Levi, "Binary dynamics from spin1-spin2 coupling at fourth post-Newtonian order," Phys. Rev. D85 (2012) 064043, arXiv:1107.4322 [gr-qc].

37. R. A. Porto and I. Z. Rothstein, "Calculation of the First Nonlinear Contribution to the General-Relativistic Spin-Spin Interaction for Binary Systems," Phys. Rev. Lett. 97 (2006) 021101, arXiv:gr-qc/0604099 [gr-qc].

38. S. Hergt and G. Schäfer, "Higher-order-in-spin interaction Hamiltonians for binary black holes from source terms of Kerr geometry in approximate ADM coordinates," Phys. Rev. D77 (2008) 104001, arXiv:0712.1515 [gr-qc].

39. S. Hergt and G. Schäfer, "Higher-order-in-spin interaction Hamiltonians for binary black holes from Poincare invariance," Phys. Rev. D78 (2008) 124004, arXiv:0809.2208 [gr-qc].

40. M. Levi and J. Steinhoff, "Leading order finite size effects with spins for inspiralling compact binaries," JHEP 06 (2015) 059, arXiv: 1410.2601 [gr-qc].

41. S. Marsat, "Cubic order spin effects in the dynamics and gravitational wave energy flux of compact object binaries," Class. Quant. Grav. 32 (2015) 085008, arXiv: 1411.4118 [gr-qc].

42. V. Vaidya, "Gravitational spin Hamiltonians from the S matrix," Phys. Rev. D91 (2015) 024017, arXiv:1410.5348 [hep-th].

43. L. Blanchet, "Gravitational Radiation from Post-Newtonian Sources and Inspiralling Compact Binaries," Living Rev. Rel. 17 (2014) 2, arXiv:1310.1528 [gr-qc].

44. J. Steinhoff, "Canonical formulation of spin in general relativity," Annalen Phys. 523 (2011) 296-353, arXiv:1106.4203 [gr-qc].

45. S. Hergt, J. Steinhoff, and G. Schäfer, "Elimination of the spin supplementary condition in the effective field theory approach to the post-Newtonian approximation," Annals Phys. 327 (2012) 1494-1537, arXiv:1110. 2094 [gr-qc].

46. M. Levi and J. Steinhoff, "Equivalence of ADM Hamiltonian and Effective Field Theory approaches at next-to-next-to-leading order spin1-spin2 coupling of binary inspirals," JCAP 1412 (2014) 003, arXiv:1408.5762 [gr-qc].

47. J. Steinhoff, "Spin and quadrupole contributions to the motion of astrophysical binaries," Fund. Theor. Phys. 179 (2015) 615-649, arXiv: 1412.3251 [gr-qc] .

48. J. Steinhoff, "Spin gauge symmetry in the action principle for classical relativistic particles," arXiv:1501.04951 [gr-qc].

49. G. N. Fleming, "Covariant position operators, spin, and locality," Phys. Rev. 137 (1965) B188-B197. 
50. K. Yagi and N. Yunes, "I-Love-Q," Science 341 (2013) 365-368, arXiv: 1302.4499 [gr-qc].

51. K. Yagi and N. Yunes, "I-Love-Q Relations in Neutron Stars and their Applications to Astrophysics, Gravitational Waves and Fundamental Physics," Phys. Rev. D88 (2013) 023009, arXiv:1303.1528 [gr-qc].

52. G. Pappas and T. A. Apostolatos, "Effectively universal behavior of rotating neutron stars in general relativity makes them even simpler than their Newtonian counterparts," Phys. Rev. Lett. 112 (2014) 121101, arXiv: 1311.5508 [gr-qc].

53. S. Chakrabarti, T. Delsate, N. Gürlebeck, and J. Steinhoff, "I-Q relation for rapidly rotating neutron stars," Phys. Rev. Lett. 112 (2014) 201102, arXiv:1311.6509 [gr-qc].

54. K. Yagi, K. Kyutoku, G. Pappas, N. Yunes, and T. A. Apostolatos, "Effective No-Hair Relations for Neutron Stars and Quark Stars: Relativistic Results," Phys. Rev. D89 (2014) 124013, arXiv:1403.6243 [gr-qc]. 